\title{
The Diagnostic and \\ Prognostic Role of Serum MicroRNAs in Ovarian \\ Cancer
}

\author{
Yin Min (First author) \\ Medical College of Qingdao University, \\ Qingdao, Shandong Province, China \\ Chen Aiping (Corresponding author) \\ Department of Gynecology, The Affiliated Hospital of Qingdao University, \\ Qingdao, Shandong Province, China
}

Received: June 20, 2018 Accepted: July 6, 2018

doi:10.5296/jbls.v9i2.13551 URL: https://doi.org/10.5296/jbls.v9i2.13551

\begin{abstract}
Aim

Summarize the research progress of serum miRNAs in clinical application for early diagnosis and prognosis of ovarian cancer.

Methods

Review of the recently published literature was conducted. We selected reviews and clinical researches from different scientific societies.

Results

The abnormal expression of serum miRNAs has a certain clinical value in the

early diagnosis of ovarian cancer. Many miRNAs, which are involved in regulating ovarian cancer cell growth, migration, and drug resistance, are also closely related to the patients' survival rate and affect the prognostic condition of the patients. So the serum miRNAs may be useful diagnostic and prognostic biomarkers.
\end{abstract}


Conclusions

Studies have shown that serum miRNAs has the tumor specificity and high stability, enabling it has the potential for early diagnosis, prognosis and sensitivity to chemotherapy. However, the researches on serum miRNAs is still at the primary stage, further researches is needed for application of serum miRNAs in early diagnosis and prognosis of ovarian cancer.

Keywords: microRNAs; ovarian cancer; serum; early detection

\section{Introduction}

Ovarian cancer is one of the most common gynecologic malignancies, whose mortality rate is the highest among gynecologic tumors. If diagnosed at early stage, the five-year survival rate is estimated to be as high as $90 \%$. Although treatments of surgical tumor debulking and platinum- and taxane- based chemotherapy was administrated, the five-year survival rate for patients with advanced ovarian cancer is still only $30 \%$ (Siegel RL et al.,2016). The lack of early detection methods for ovarian cancer is considered as one of the reasons for its high mortality rate. Currently, diagnosis methods may involve pelvic examination, imaging examination (transvaginal ultrasound, CT, MRI) and tumor biomarkers, including carbohydrate antigen 125 (CA125), human epididymis protein(HE4). But the sensitivity and specificity of these tests or examinations are low, a majority of patients failed to get the early diagnosis and treatment, so it is necessary to search for new approaches for detecting ovarian cancer at early stage. miRNAs are a class of non-coding endogenous RNAs that are typically 19-25 nucleotides in length, participating in post-transcriptionally regulation of genes(Bartel DP et al.,2004).Studies have indicated that miRNAs can regulate nearly $60 \%$ of human gene expression, which are involved in crucial biological processes such as cellular proliferation, differentiation, cell cycle control, and apoptosis(Berezikov E et al.,2005). miRNA does not only exist in the cytoplasm but also in many types of body fluids including blood, ascites and urine. Cell-free miRNAs in circulating blood has been shown to be useful in the early diagnosis and prognosis of cancers, as well as in the response to chemotherapy (Schwarzenbach et al.,2014).This review focuses on the clinical role and future trends of serum miRNAs in the diagnosis and prognosis evaluation of ovarian cancer.

\section{2. miRNAs and Ovarian Cancer}

miRNAs are small single-stranded non-coding RNAs in a length of about 19-25 nucleotides. By base-pairing to the untranslated region of the target mRNA, miRNAs show the effect of gene expression modulation, resulting in either translational repression, mRNA cleavage, or destabilization(Bartel DP et al.,2004). About 2500 kinds of human miRNAs was found, and miRNAs can control more than half of the genes concerning a variety of cell activities. What's more, many studies have shown that miRNAs may play a role in cacinogenesis and cancer progression by modulating the expression of genes related to cell cycle, cell differentiation, apoptosis, distant metastasis and angiogenesis(He L et al.,2004). The expression profile of miRNA varies in different tumors, and certain miRNAs are up-regulated or down-regulated, showing a function of oncogenes or tumor suppressor genes 
(Esquela-Kerscher A et al.,2006).Compared with normal or benign ovarian tissue, the expression of miRNAs in ovarian cancer is often abnormal. Iorio et al. conducted a research and showed different miRNAs expression profiles between ovarian cancer tissues and normal tissues. Among twenty nine detected miRNAs, they found that only miR-141, miR-200a, miR-200b, and miR-200c were up-regulated and twenty five were down-regulated in the cancer samples. They also found that miRNAs signatures vary in different ovarian carcinoma histotypes(Iorio MV et al.,2007). Additionally, it has been shown that miRNAs can also be histotype-specific. Calura et al. aimed to identify subtype-specific biomarkers. Specifically, the clear cell histotype showed a higher expression of miR-30a and miR-30a, whereas miR-192/194 were up-regulated in mucinous histotype. Ohyagi-Hara et al. found that the over expression of miR-92a in ovarian cancer cells markedly suppressed peritoneal dissemination in vivo, indicating that miR-92a may prove to be a novel target for gene therapy (Ohyagi-Hara C et al.,2013). These studies suggest that miRNAs may be involved in the development and progression of ovarian cancer. They often affect the biological behaviors of ovarian cancer such as growth, invasion, and metastasis by modulating the downstream target genes.

\section{3. miRNAs in Serum}

According to the past opinions, as a large amount of endogenous nucleases were found in the body fluid, the cell-free circulating RNA was degraded rapidly. However, recent evidences suggest that the secreted miRNAs from cells into circulation can stably exist(Kosaka $\mathrm{N}$ et al.,2010). Since miRNAs in serum and plasma detected for the first time in 2008, they were found in various types of body fluids, such as breast milk, saliva, urine and so on. So far several researches have shown that the level of miRNAs in serum is consistent with that in tissues, enabling serum miRNAs potential biomarkers of cancer(Chung $\mathrm{Y}$ et al,.2013).Lawrie et al. first described serum miRNAs in three patients with cancer and suggested that miRNAs have potential to be used as a noninvasive diagnostic marker for diffuse large B cell lymphoma and other tumors (Lawrie CH et al.,2008). Serum miRNAs as a tumor biomarker relies on their high stability and the ability to reflect tumor status and predict the response to chemotherapy. It was reported that circulating miRNAs could be stable in harsh conditions such as repetitive freeze-thaw cycles, extreme $\mathrm{pH}$, prolonged storage time, and boiling(Mitchell PS et al.,2008). The incredible stability can be attributed to proteins complexes like Ago2 and circulating micro vesicles such as exosomes. Arroyo et al. found that most circulating miRNAs in plasma are cofractionated with Ago2, indicating that circulating Ago2 complexes may contribute to the stability of plasma miRNA (Arroyo JD et al.,2011). It was also reported that high-density lipoprotein (HDL) can transport endogenous miRNAs, delivering them to target cells, according to the study from Vickers(Vickers KC et al.,2011). Wittmann et al. have shown that the expression profiles of miRNAs varies indifferent physiological and pathological conditions (Wittmann J et al.,2010). The stability and specificity strongly support the idea that circulating serum miRNAs concerning tumor have the potential to become valuable tumor biomarkers(Gross WL et al.,2014). 


\section{Serum miRNAs and ovarian cancer}

Currently, CA125 is one of the major biomarker for the early diagnosis and prognosis of ovarian cancer. However, CA125 has many limitations in the diagnosis of ovarian cancer. Firstly, its diagnostic sensitivity is low, so it cannot realize the early detection on small and curable cancer. In addition, its diagnostic specificity is poor. CA125 is increased to different degrees in other conditions including pregnancy, menstruation and endometriosis, as well as other cancers, so there are some false positives. A study with 118 ovarian cancer patients, 84 patients with benign ovarian diseases and 61 healthy women showed that the sensitivity and specificity of CA125 were $77.4 \%$ and $70.8 \%$ ( Kim Y W et al.,2012), respectively. So there is an urgent need to find a better biomarker for early diagnosis of ovarian cancer effectively. Serum, as a kind of biomarker, is a hot research topic. Compared to the rest of biomarkers, serum is equipped with the advantages of convenience, feasibility, less-invasiveness, and continuous detection in vitro. Zheng et al. found the high stability of serum miRNAs which can be not affected by RNase, $\mathrm{pH}$ and low temperature environment. The high stability also enables the measured results to be in accordance with the actual level roughly(Zhenggang et al.,2011). Therefore, serum miRNAs has unique advantages(Kan C W et al.,2012).

\subsection{Potential of serum miRNAs as diagnostic biomarkers}

In recent years, more and more researches on the diagnostic potential of circulating miRNAs in ovarian cancervhas been conducted. As early as 2008, Taylor et al. reported the miRNAs signatures of tumor-derived exosomes and compared miRNAs expression profiles between benign controls and ovarian cancer patients. Eight miRNAs were found to be overexpressed in ovarian cancer patients, even in patients with early stage. Thus, these 8 target miRNAs in peripheral blood could be used as potential biomarkers for screening patients without apparent symptoms of ovarian cancer(Taylor D D et al.,2008). Resnick et al. compared twenty one serum miRNAs between healthy controls and epithelial ovarian cancer patients(Resnick K E et al.,2008). MiR-21, miR-92 and miR-93 were found to be higher in ovarian cancer patients with normal CA125, suggesting miRNAs could be a biomarker for early diagnosis in patients with normal CA125 levels. Kan et al. investigated the levels of 4 miRNAs (miR-200a, b, c and miR-182) in the serum of patients with serous epithelial ovarian cancer( SEOC) and healthy controls. The miR-200 family were significantly elevated. And a combination of miR-200b and miR-200c showed the best predictive power to discriminate SEOC and healthy serum, with a ROC-AUC of 0.784, suggesting that the addition of these microRNAs to current testing regimes may improve diagnosis for women with SEOC(Kan C W et al.,2012). Meng et al. reported that serum levels of miR-25 and miR-93 in patients were downregulated, whereas those of miR-7 and miR-429 were upregulated compared with healthy women. The sensitivity and specificity of these four microRNAs to discriminate EOC patients from healthy women can reach to $93 \%$ and92\%( Meng X et al.,2015). In their later study, the serum concentrations of cell-free miR-373, miR-200a, miR-200b and miR-200c showed significantly higher in EOC patients than in healthy women. Consequently, the combination of miR-200a, miR-200b and 
miR-200c could differ between malignant and benign ovarian tumors, with a sensitivity of $83 \%$ and a specificity of $100 \%$. Therefore the increased serum levels of this microRNAs panel can act as a diagnostic tool to distinguish healthy controls and benign tumors from EOC( Meng X et al.,2015). As for different histotypes, Chao A et al. evaluated serum samples of twenty-one patients with clear cell carcinoma. Four miRNAs (hsa-miR-130a, hsa-miR-138, hsa-miR-187, and hsa-miR-202) were confirmed to be higher in the preoperative sera. More importantly, in three patients, hsa-miR-130a levels were elevated in early disease recurrences before CA125 was found to be elevated(Chao A et al.,2014). Zuberi et al. revealed that serum miR-200a overexpression was found be associated with tumor histology and stage(Zuberi Met al.,2015).

Above all, the abnormal expression of serum miRNAs has a certain clinical value in the early diagnosis of ovarian cancer.

\subsection{Prognostic potential of serum miRNAs in ovarian cancer}

More and more studies have found that the expression level of miRNAs in serum can be used not only in the diagnosis of ovarian cancer, but also as an important tool for assessing the prognosis of patients. Many miRNAs, which are involved in regulating ovarian cancer cell growth, migration, and drug resistance, are also closely related to the patient's survival rate and affect the prognostic condition of the patients.

$\mathrm{Xu}$ et al. demonstrated that serum miR-21 levels in EOC patients were significantly higher than in healthy controls. In addition, increased serum miR-21 expression was correlated with high tumor grade, advanced FIGO stage, and shortened overall survival showing that high-level of serum miR-21 expression was an unfavorable prognostic factor independent of other clinicopathological factors(Xu Y Z et al.,2013). Gao et al. found the relative expression level of miR-141 displayed an escalating trend, while miR-200c showed a descending trend from early stages to advanced stages. Furthermore, patients with high miR-200c level achieved significantly a higher 2-year survival rate compared with the other group, while low miR-141 group showed a significantly higher survival rate(Gao Y C et al.,2015). Meng et al. found that the levels of miR-429 positively correlated with CA125 values and differed between FIGO I-II and III-IV stages. In addition, overexpressed miR-7 in SKOV3 cells resulted in increased cell migration and invasion( Meng X et al.,2015). Guo et al. evaluated the levels of serum miR-92 in patients with epithelial ovarian cancer with lymph node metastasis, and found the expression level increased significantly compared with that without lymph node metastasis. It was also upregulated significantly in the earlier stage. So we can evaluate the disease progression by measuring serum miR-92 level(Fengjun $G$ et al.,2013).

At present,the standard treatment of ovarian cancer is the surgical tumor reduction maximally plus platinum and paclitaxel combined chemotherapy. Although the initial response rate to chemotherapy can be as high as70\%,subsequent relapses and repeated cytotoxic chemotherapies eventually lead to acquired resistance to the treatments, so the 5-years survival rate is less than 40\%( Frederick P J et al.,2013). This high mortality rate can be attribute to the resistance to chemotherapy, especially in recurrent cases. Therefore, 
researches in this field are in great need in order to find effective predictive and prognostic markers to get optimal and personal treatment of ovarian cancer. MiRNAs can modulate expression of multiple genes relating to drug sensitivity. And the expression level of miRNAs in serum is parallel to that of tissue, which can be used to assess the individual resistance to chemotherapy. Therefore individualized adjustments can be made to achieve the best curative effect and improve the prognosis of patients. Feng et al. conducted a study on the correlation between serum miRNAs and cisplatin sensitivity in ovarian cancer patients and found that miR-22 in peripheral blood was significantly upregulated(about 3.2 times higher) in patients after chemotherapy. Additionally patients with higher level of miR-22 showed more sensitive response to chemotherapy, suggesting serum miR-22 and sensitivity of chemotherapy was positively correlated(Zhaoxia F et al.,2016). Lang he et al. found that early loss of miR-let-7i-5P in serum of ovarian cancer patients is prone to chemotherapy resistance, suggesting that serum miR-let-7i-5p is one of the prognostic markers of ovarian cancer (Zuberi M et al.,2016). Satoshhi et al. found that forced expression of miR-135a-3p in SKOV-3 and ES-2 human ovarian cancer cells induced drug sensitivity to cisplatin and paclitaxel and suppressed cell proliferation and xeno-graft tumor growth. These findings supported the idea that miR-135a-3p may be considered as a biomarker and a therapeutic agent in ovarian cancer(Fukagawa $\mathrm{S}$ et al.,2017).

\section{Conclusion}

The pathogenesis of ovarian cancer is not completely understood to date. Although pelvic examination, ultrasound, CA125, and HE4 remain to be the major approaches for ovarian cancer diagnosis, their low sensitivity, specificity and invasiveness hinder most of their clinical use. With the development of the biology of circulating nucleic acids and powerful technologies for their analysis, our understanding for circulating cell-free miRNAs has greatly progressed. Consequently, more and more studies have shown that serum miRNA has the tumor specificity and high stability, enabling it has the potential for early diagnosis, prognosis and sensitivity to chemotherapy. However, the researches on serum miRNA is still at the primary stage, the existing research still has some limitations: the sample size is relatively small; the level of serum miRNA of patients was simply detected to change, lacking of clinical effectiveness comparison with traditional diagnostic methods such as CA125 and ultrasound; there is no standardized testing procedures, including sample collection, RNA extraction, selecting appropriate internal control. Therefore, further researches are still needed on how to select stable and reliable specific miRNA profiles, how to formulate effective scheme of combined detection of miRNA. Detailed understandings of the serum miRNA of ovarian cancer patients could contribute to novel approaches in early diagnosis, prognosis, and personalized therapies and so on, thus bringing new progress for ovarian cancer.

\section{Acknowledgement}

The authors would like to acknowledge the gynaecology teams in The Affiliated Hospital of Qingdao University, Qingdao, Shandong Province, China. We also thank Bai Yiqi for editorial assistance and emotional encouragements. 


\section{References}

Bartel DP. MicroRNAs: genomics, biogenesis, mechanism, and function[J]. Cell. 2004,116(2):281-97.

Berezikov E, Guryev V, Van d B J, et al. Phylogenetic shadowing and computational identification of human microRNA genes[J]. Cell, 2005, 120(1):21-4.

Chao A, Lai C H, Chen H C, et al. Serum microRNAs in clear cell carcinoma of the ovary[J]. Taiwanese Journal of Obstetrics \& Gynecology, 2014, 53(4):536-41.

Chung Y W, Bae H S, Song J Y, et al. Detection of microRNA as novel biomarkers of epithelial ovarian cancer from the serum of ovarian cancer patients.[J]. International Journal of Gynecological Cancer Official Journal of the International Gynecological Cancer Society, 2013, 23(4):673-9.

Esquela-Kerscher A, Slack F J. Esquela-Kerscher, A and Slack, FJ. Oncomirs-microRNAs with a role in cancer. Nat Rev Cancer 6: 259-269[J]. Nature Reviews Cancer, 2006, 6(4):259-269.

Fengjun G, Jingyan T, Yang L, et al. Serum microRNA-92 expression in patients with ovarian epithelial carcinoma[J]. Journal of International Medical Research, 2013, 41(5):1456.

Frederick P J, Green H N, Huang J S, et al. Chemoresistance in ovarian cancer linked to expression of microRNAs.[J]. Biotechnic \& Histochemistry, 2013, 88(7):403-409.

Fukagawa S, Miyata K, Yotsumoto F, et al. MiR-135a-3p as a promising biomarker and nucleic acid therapeutic agent for ovarian cancer[J]. Cancer Science, 2017, 108(5):886-896.

Gao Y C, Wu J. MicroRNA-200c and microRNA-141 as potential diagnostic and prognostic biomarkers for ovarian cancer[J]. Tumour Biology the Journal of the International Society for Oncodevelopmental Biology \& Medicine, 2015, 36(6):4843.

Gross WL, Hauschild S, Mistry N. Clinical relevance of circulating cell-free microRNAs in cancer[J]. Nature Reviews Clinical Oncology. 2014,11(3):145-56.

He L, Hannon G J. MicroRNAs: small RNAs with a big role in gene regulation[J]. Nature Reviews Genetics, 2004, 5(7):522.

Iorio MV, Visone R, Di Leva G, et al. MicroRNA signatures in human ovarian cancer[J]. Cancer research. 2007,67(18):8699-707.

Kan C W, Hahn M A, Gard G B, et al. Elevated levels of circulating microRNA-200 family members correlate with serous epithelial ovarian cancer[J]. BMC Cancer, 2012, 12(1):627.

Kosaka N, Iguchi H, Yoshioka Y, et al. Secretory mechanisms and intercellular transfer of microRNAs in living cells[J]. The Journal of biological chemistry. 2010,285(23):17442-52.

Kim Y W, Su M B, Lim H, et al. Development of Multiplexed Bead-Based Immunoassays for the Detection of Early Stage Ovarian Cancer Using a Combination of Serum Biomarkers[J]. Plos One, 2012, 7(9):e44960. 


\section{MInstitute Macrothink $_{\text {Int }}$}

Langhe R, Norris L, Saadeh F A, et al. A novel serum microRNA panel to discriminate benign from malignant ovarian disease[J]. Cancer Letters, 2015, 356(2):628-636.

Lawrie CH, Gal S, Dunlop HM, et al. Detection of elevated levels of tumour-associated microRNAs in serum of patients with diffuse large B-cell lymphoma[J]. British journal of haematology. 2008,141(5):672-5.

Meng X, Joosse SA, Müller V, et al. Diagnostic and prognostic potential of serum miR-7, miR-16, miR-25, miR-93,miR-182, miR-376a and miR-429 in ovarian cancer patients. British Journal of Cancer.2015; 113:1358-66.

Mitchell PS, Parkin RK, Kroh EM, et al. Circulating microRNAs as stable blood-based markers for cancer detection[J]. Proceedings of the National Academy of Sciences of the United States of America. 2008,105(30):10513-8.

Ohyagi-Hara C, Sawada K, Kamiura S, et al. miR-92a inhibits peritoneal dissemination of ovarian cancer cells by inhibiting integrin alpha5 expression[J]. The American journal of pathology. 2013,182(5):1876-89.

Resnick K E, Alder H, Hagan J P, et al. The detection of differentially expressed microRNAs from the serum of ovarian cancer patients using a novel real-time PCR platform. [J] Gynecologic Oncology, 2008, 112(1):55-59.

Schwarzenbach H, Nishida N, Calin GA, et al. Clinical relevance of circulating cell-free microRNAs in cancer[J]. Nature reviews Clinical oncology. 2014,11(3):145-56.

Siegel RL, Miller KD, Jemal A. Cancer statistics, 2016. CA: a cancer journal for clinicians. 2016,66(1):7-30.

Vickers KC, Palmisano BT, Shoucri BM, et al. MicroRNAs are transported in plasma and delivered to recipient cells by high-density lipoproteins[J]. Nature Cell Biology. 2011,17(1):423-33.

Wittmann J, Jäck HM. Serum microRNAs as powerful cancer biomarkers[J]. Biochimica Et Biophysica Acta. 2010,1806(2):200-7.

Xu Y Z, Xi Q H, Ge W L, et al. Identification of serum microRNA-21 as a biomarker for early detection and prognosis in human epithelial ovarian cancer.[J]. Asian Pacific Journal of Cancer Prevention Apjcp, 2013, 14(2):1057.

Zhenggang, Jiang, Lijian, et al. Stability analysis of liver cancer-related microRNAs[J]. Acta Biochimica Et Biophysica Sinica, 2011, 43(1):69-78.

Zuberi M, Mir R, Das J, et al. Expression of serum miR-200a, miR-200b, and miR-200c as candidate biomarkers in epithelial ovarian cancer and their association with clinicopathological features[J]. Clinical and Translational Oncology, 2015, 17(10):1-9.

Zuberi M, Khan I, Gandhi G, et al. The conglomeration of diagnostic, prognostic and therapeutic potential of serum miR-199a and its association with clinicopathological features in epithelial ovarian cancer[J]. Tumor Biology, 2016, 37(8):11259-11266. 


\section{Macrothink}

Zhaoxia F, Yuliang H, Xianhui OY,et al. Study of the relationship between miRNA level in blood plasma of ovarian cancer patients and chemotherapy sensitivity to cis-platin.Journal of Chinese Physician 16(7):930-933.

\section{Copyright Disclaimer}

Copyright for this article is retained by the author(s), with first publication rights granted to the journal.

This is an open-access article distributed under the terms and conditions of the Creative Commons Attribution license (http://creativecommons.org/licenses/by/3.0/). 